\title{
Factors Associated with Sample Rejection for CD4+/CD8+ T Cell Count Analyses at the Kenyatta National Hospital Comprehensive Care Center Laboratory, Kenya
}

\author{
Moherai Wilfred Felix ${ }^{1}$, Joshua Nyagol ${ }^{*}$, Walter Mwanda ${ }^{2}$ \\ ${ }^{1}$ Department of Human Pathology, Thematic Unit of Immunology, University of Nairobi, Nairobi, Kenya \\ ${ }^{2}$ Department of Human Pathology, Thematic Unit of Hematology and Blood Transfusion Unit, University of Nairobi, Nairobi, Kenya \\ Email: ^jnyagol133@gmail.com
}

How to cite this paper: Felix, M.W., Nyagol, J. and Mwanda, W. (2021) Factors Associated with Sample Rejection for CD4+/ CD8+ T Cell Count Analyses at the Kenyatta National Hospital Comprehensive Care Center Laboratory, Kenya. World Journal of AIDS, 11, 181-188.

https://doi.org/10.4236/wja.2021.114013

Received: October 20, 2021

Accepted: December 25, 2021

Published: December 28, 2021

Copyright $\odot 2021$ by author(s) and Scientific Research Publishing Inc. This work is licensed under the Creative Commons Attribution International License (CC BY 4.0).

http://creativecommons.org/licenses/by/4.0/

\begin{abstract}
Background: The appropriate time to initiate antiretroviral therapy (ART) in HIV/AIDS patients is determined by measurement of CD4+/CD8+ T cell count. The CD4/CD8+ T cell count is also useful, together with viral load, in monitoring disease progression and effectiveness treatment regimens. Several factors may contribute to sample rejection during the CD4+/CD8+ T cells count, resulting in negative effects on patient management. Objective: Evaluate the causes for $\mathrm{CD} 4+\mathrm{CD} 8+\mathrm{T}$ cell count sample rejection at the Kenyatta National Hospital Comprehensive Care Center Laboratory. Method: A retrospective cross-sectional study was conducted between 2018 and 2020. Data was obtained from the "rejected samples" for Partec ${ }^{\mathrm{R}}$ FlowCyp flow cytometry file. Designed data collection sheet was used for data capture. A total of 3972 samples were submitted for CD4+/CD8+ T cell count during the study period. Causes for sample rejection were numbered 1 to 12, each representing a reason for sample rejection. Number 1 was sub-categorized into clotted, hemolyzed, short-draw and lipemic. Data was analyzed using excel, and presented using tables, graphs and pie charts. Approval to conduct the study was obtained from KNH/UoN ERC. Results. In the study period, 81/3972 (2.0\%) samples were rejected. Samples submitted more than 48 hours after collection were mostly rejected. Other factors included improper collection technique, delayed testing, patient identification error and incorrect use of vacutainer. A combination of clotted samples, specimen submission more than 48 hours caused the most frequent sample rejection, followed with combination of specimen submission more than 48 hours, delayed testing and delayed specimen processing. Together, clotted samples, incorrect vacutainer and poor specimen label caused the least sample rejection. Conclusion: Sample rejection
\end{abstract}


rate for $\mathrm{CD} 4 / \mathrm{CD} 8+\mathrm{T}$ cell count was relatively low, and multiple factors contributed to rejection. However, improved quality assurance will enable more benefit to patients who seek this test in the laboratory.

\section{Keywords}

Sample, Rejection, Causes, CD4/CD8+ T Cell Count, Flow Cytometry

\section{Introduction}

During HIV/AIDS disease progression, it is important for the care providers to have knowledge of $\mathrm{CD} 4 / \mathrm{CD} 8+\mathrm{T}$ cells count of their patients. This has been possible due, in part, to improved HIV diagnosis, complemented with care and treatment [1]. Two critical factors determine reliability of sample testing in a referral laboratory. Firstly, the established quality assurance must ensure accurate results, and secondly, turnaround time that translates to timely release of results must be guaranteed. In HIV/AIDS management, the disease outcome may be determined by suitability of the sample collected for the analyses requested. Sample rejection during the $\mathrm{CD} 4 / \mathrm{CD} 8+\mathrm{T}$ cell count presents a challenge in many laboratories, and may impact negatively on the desired treatment outcome in these patients [2] [3].

Sample acquisition and preanalytical stages constitute critical steps in laboratory medicine, and involve request for a test, patient identification, sample collection and transport, and sample preparation for analysis [4]. Several studies have reported that nearly $70 \%$ of errors that lead to sample rejection could occur at the preanalytical stage [3] [5] [6].

Criterion for sample rejection has been described to constitute a list of factors which classify a sample as being unfit for analysis. These could range from improper sample collection, inappropriate sample type, incorrect labelling, to poor sample transport, and improper storage [7]. Although some of these reasons may vary, depending on the type of tests being assayed, a number of these causes for sample rejection do apply across board, and do not depend on the test being requested. For instance, sample storage is dependent on the test to be done, while improper sample labelling may not be a test-specific criterion for any laboratory sample rejection.

Currently, enumeration of CD4/CD8 + T cells in HIV/AIDS management is performed using flow cytometry technique. It remains a powerful tool to analyze the physical and biological properties of a cell in a short period of time, and uses dyes or monoclonal antibodies that target molecules located either on the surface or inside of the cell [8]. At Kenyatta National Hospital Comprehensive Care Center (KNH-CCC) Laboratory, the demand for CD4/CD8+ T cell count has expanded enormously in the recent past, as it serves both as in-patients and referral laboratory testing for HIV/AIDS patients on ART management. This presents with a challenge in achieving efficacy in ART, particularly due to sam- 
ple rejection during the CD4/CD8 + T cell count in a section of samples analyzed in the laboratory, typical to referral laboratories [9] [10].

The present study sought to identify the common causes of sample rejection for CD4/CD8+ T cell flow cytometry analysis, to guide on improvement in service provision for quality laboratory results and minimize patient mismanagement.

\section{Methods}

\subsection{Ethical Approval}

Approval to conduct the study was obtained from KNH/UoN ERC. There was no contact with the patients, and request for exemption for informed signed consent was included in the application for ethical approval. All procedures performed in the current study were approved by $\mathrm{KNH} / \mathrm{UoN}$ research ethics committee (reference number UP313/05/2021, and dated $07^{\text {th }}$ May, 2021), in accordance with the 1964 Helsinki declaration and its later amendments.

\subsection{Study Design, Area and Population}

This was a retrospective cross-sectional study carried out at Kenyatta National Hospital, Comprehensive Care Center Laboratory. The laboratory mainly tests samples for HIV/AIDS patients presenting to the hospital, as well as samples submitted from referral health care facilities. The laboratory has five sections, namely; Flow Cytometry, Molecular, Biochemistry, Hematology and TB GeneXpert. This study was done in the flow cytometry section, with all the samples rejected at the flow cytometry section from the year 2018 to 2020 included.

\subsection{Data Collection}

Data on sample rejection in the laboratory was extracted using designed data collection sheets. The data collected included the causes of sample rejection and frequency of rejection for any individual or multiple causes. Each sample rejection form was given an identification research number for the study. Patients' identification data were de-identified. The data was then cleaned and stored using Python. To protect the privacy of the patients whose samples were analysed, the stored data was password protected, and only accessible to authorized principal investigator and the supervisor.

\subsection{Data Analysis}

The data was analyzed using Microsoft excel and rapid miners, and presented in tables, charts and graphs.

\section{Results}

\subsection{Causes of Sample Rejection}

In the study period, 3972 samples were submitted for CD4/CD8+ T cell count, out of which 81 were rejected, representing rejection rate of $2.0 \%$. Several factors were documented to cause sample rejection as shown in Table 1. 
Table 1. Causes of sample rejection for CD4+/CD8+ T cell analyses.

\begin{tabular}{cc}
\hline Cause(s) & Number of samples rejected (\%) \\
\hline Improper collection technique & $11(13.58)$ \\
Incorrect container/tube & $3(3.70)$ \\
Patient ID error & $5(6.17)$ \\
Flow cytometry specimen $>48$ hours & $28(34.57)$ \\
Delayed testing & $10(12.35)$ \\
Delayed specimen processing & $9(11.11)$ \\
Other causes & $15(18.52)$ \\
\hline
\end{tabular}

\subsection{Multiple Causes for Sample Rejection}

Multiple causes for rejection for a single sample were also analyzed. In this case, the causes were assigned numbers $1-12$, with number 1 being subcategorized further into $\{$ i\} clotted. \{ii\} hemolyzed. \{iii\} short-draw. $\{$ iv $\}$ lipemic, as shown below, also shown in Table 2 and Figure 1.

Key (causes for rejection);

1) Improper collection technique:

\{i\} clotted \{ii\} hemolyzed \{iii\} short-draw \{iv\} lipemic.

2) Incorrect container/tube received.

3) Patient ID error/no specimen label.

4) Requisition and Specimen do not match.

5) Improperly packaged specimen.

6) Delayed specimen delivery.

7) Specimens with the same ID.

8) Flow cytometry specimen $>48 \mathrm{~h}$.

9) Delayed testing.

10) Delayed specimen processing.

11) No specimen requisition form.

12) Other cause(s).

\section{Discussion}

The Kenyatta National Hospital Comprehensive Care Laboratory, Flow Cytometry Section, uses a high through-put cytometer, which is supported with both quality control and external quality assurance in test performance. This study found out that 81 out of 3972 samples received for CD4/CD8+ T cells counts between 2018 and 2020 were rejected, reflecting a rejection rate of $2.0 \%$. Arguably, this implies that disease progression in HIV/AIDS, and management intervention through ART could not benefit the patients whose samples did not meet the threshold for CD4/CD8+ T cell count analyses. Similar challenges have been reported in previous studies [10] [11]. 
Table 2. Multiple causes of sample rejection.

\begin{tabular}{cc}
\hline Multiple causes as keyed above & Number of samples rejected (\%) \\
\hline $8,9,10,12$ & $4(4.94)$ \\
$1\{\mathrm{i}\}, 8,12$ & $8(9.88)$ \\
$1\{\mathrm{i}\}, 2,3$ & $1(1.23)$ \\
$4,8,9$ & $2(2.47)$ \\
$8,9,10$ & $7(8.64)$ \\
8,9 & $3(3.70)$ \\
8,10 & $2(2.47)$ \\
$8,9,10,11$ & $4(4.94)$ \\
\hline
\end{tabular}

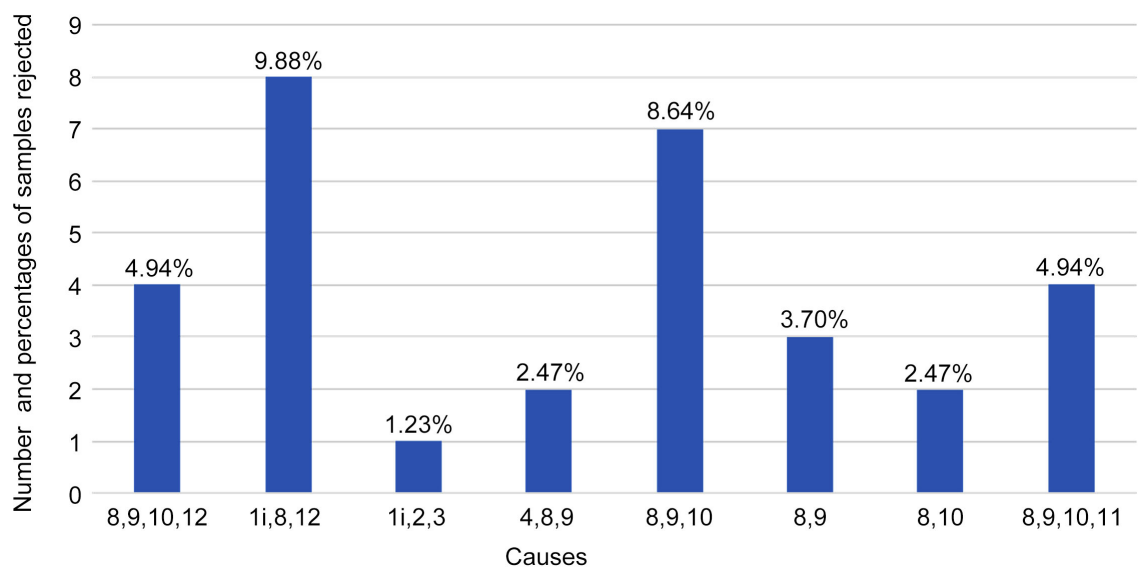

Figure 1. Combination of causes for sample rejection for CD4+/CD8+ T cell analyses.

Many peripheral laboratories have low capacity in CD4/CD8+ $\mathrm{T}$ cell testing for HIV/AIDS patients in low- and middle-income countries, and Kenya is not an exception. Thus, the referral laboratories have the burden to meet the standards of the test performance, while adhering to protocols for quality results [12] [13]. Many patients at times must travel long distances for this essential testing or have their specimens transported to KNH-CCC laboratory. Consequently, samples that stay beyond forty-eight hours before analyses will be deemed unsuitable for $\mathrm{CD} 4 / \mathrm{CD} 8+\mathrm{T}$ cell counts. Although not confined to $\mathrm{KNH}-\mathrm{CCC}$ laboratory, other contributory factors in sample rejection during $\mathrm{CD} 4 / \mathrm{CD} 8+\mathrm{T}$ cell counts have been reported in other studies. These include skilled human resource limitations, poor laboratory infrastructure and lack of laboratory information management system that ensures quality testing [14] [15].

In the present study, twelve criteria for sample rejection were determined, from which it was established that samples were rejected for seven reasons. These were: improper collection technique-clotted sample (11 samples, $13.5 \%)$, incorrect container/tube received ( 3 samples, 3.7\%), patient ID error/no specimen label ( 5 samples, $6.1 \%$ ), flow cytometry specimen stayed more than 48 hours before analysis (28 samples, 34.5\%), delayed testing (10 samples, 12.3\%), delayed 
specimen processing ( 9 samples, $11.1 \%$ ) and unspecified reasons, only labelled as "others" in the rejection forms in the laboratory (15 samples, 18.5\%).

Notably, of the seven reasons for rejection, the most prevalent was flow cytometry specimen that had stayed more than 48 hours before analysis (28 samples, representing $34.5 \%$ ). This was mainly because some samples would arrive in the laboratory for CD4/CD8+ T cell count analyses end of the week, necessitating an overstay of the samples until the beginning of the following week. The least prevalent reason for sample rejection was incorrect container/tube received (3 samples, which represented 3.7\%). No samples were rejected for the following reasons: requisition form and specimen mismatch, improperly packaged specimen, delayed delivery of specimen, specimens with the same ID and no specimen requisition form. These findings have also been reported in other studies done elsewhere [16] [17].

Multiple causal relationship for sample rejection showed that samples could be rejected due to multiple causes. This could be explained by inter-relation of the causes for rejection. For instance, a flow cytometry specimen sample that is overstayed is also likely to be clotted thus rejected for both reasons. The most prevalent multiple reasons for rejection were improper collection technique-clotted sample, flow cytometry specimen that stayed more than 48 hours and reasons only marked as "others", and resulted in rejection of eight samples. The least prevalent multiple reasons for sample rejection were improper collection technique-clotted, incorrect container/tube received and patient ID error, and resulted in only one sample being rejected.

Our study reported that sample rejection rate at the Kenyatta National Hospital Comprehensive Care Clinic Laboratory Flow Cytometry Section may be relatively low, with the main cause of sample rejection being overstaying of samples for more than 48 hours before CD4+/CD8+ T cell count analyses. However, to eliminate the chances of sample rejection, the laboratory may improve quality measures for sample acquisition and transport to the laboratory for the benefit of the target clients.

Although other investigations were also carried out on the same samples for which rejections were reported, such as viral load, the retrospective nature of our study limited our ability to establish how the rejected samples impacted on patient management. A prospective study, analyzing many parameters in laboratory results in CD4/CD8+ T cell count for the management of HIV patients may be necessary to determine this impact.

\section{Conflicts of Interest}

The authors declare no conflicts of interest regarding the publication of this paper.

\section{References}

[1] Ceulemans, A., Bouzahzah, C., Prat, I., Urassa, W. and Kestens, L. (2019) CD4-T Cell Enumeration in Human Immunodeficiency Virus (HIV)-Infected Patients: A 
Laboratory Performance Evaluation of Muse Auto CD4/CD4\% System by World Health Organization Prequalification of in Vitro Diagnostics. PLOS ONE, 14, Article ID: e0209677. https://doi.org/10.1371/journal.pone.0209677

[2] Westerman, L.E., Kohatsu, L., Ortiz, A., McClain, B., Kaplan, J., Spira, T., et al. (2010) A Quality Management Systems Approach for CD4 Testing in Resource-Poor Settings. American Journal of Clinical Pathology, 134, 556-567. https://doi.org/10.1309/AJCPP7MCHFYLX2FM

[3] Gunnur Dikmen, Z., Pinar, A. and Akbiyik, F. (2015) Specimen Rejection in Laboratory Medicine: Necessary for Patient Safety? Biochemia Medica, 25, 377-385. https://doi.org/10.11613/BM.2015.037

[4] Plebani, M. (2012) Quality Indicators to Detect Preanalytical Errors in Laboratory Testing. Clinical Biochemist Reviews, 33, 85-88.

[5] Shiferaw, M.B., Yismaw, G. and Getachew, H. (2018) Specimen Rejections among Referred Specimens through Referral Network to the Amhara Public Health Institute for Laboratory Testing, Bahir Dar, Ethiopia. BMC Research Notes, 11, Article No. 781. https://doi.org/10.1186/s13104-018-3891-7

[6] Carraro, P. and Plebani, M. (2007) Errors in a Stat Laboratory: Types and Frequency 10 Years Later. Clinical Chemistry, 53, 1338-1342. https://doi.org/10.1373/clinchem.2007.088344

[7] International Organization for Standardization (2012) ISO 15189:2012: Medical Laboratories: Particular Requirements for Quality and Competence. International Organization for Standardization, Geneva.

[8] Adan, A., Alizada, G., Kiraz, Y., Baran, Y.N.A. (2017) Flow Cytometry: Basic Principles and Applications. Critical Reviews in Biotechnology, 37, 163-176. https://doi.org/10.3109/07388551.2015.1128876

[9] Peter, T., Badrichani, A., Wu, E., Freeman, R., Ncube, B., Ariki, F., et al. (2008) Challenges in Implementing CD4 Testing in Resource-Limited Settings. Cytometry Part B: Clinical Cytometry, 74B, S123-S130. https://doi.org/10.1002/cyto.b.20416

[10] Andrew, C. and Robyn Richardson, Z.L. (2019) Success and Failure of Initial Antiretroviral Therapy in Adults: An Updated Systematic Review. AIDS, 33, 443-453. https://doi.org/10.1097/QAD.0000000000002077

[11] Kihara, H.J. (2010) Needs Assessment for Strengthening Laboratory Human Resources Capacity for Universal Access to HIV, Malaria and TB Services in Three Sub-Saharan Countries WHO Library Cataloguing-in-Publication Data. World Health Organization, Geneva.

[12] Minchella, P.A., Chipungu, G., Kim, A.A., Sarr, A., Ali, H., Mwenda, R., et al. (2017) Specimen Origin, Type and Testing Laboratory Are Linked to Longer Turnaround Times for HIV Viral Load Testing in Malawi. PLoS ONE, 12, Article ID: e0173009. https://doi.org/10.1371/journal.pone.0173009

[13] Vitoria, M., Granich, R., Gilks, C.F, Gunneberg, C., Hosseini, M., Were, W., et al. (2009) The Global Fight Against HIV/AIDS, Tuberculosis, and Malaria: Current Status and Future Perspectives. American Journal of Clinical Pathology, 131, 844-848. https://doi.org/10.1309/AJCP5XHDB1PNAEYT

[14] Birx, D., De Souza, M. and Nkengasong, J.N. (2009) Laboratory Challenges in the Scaling up of HIV, TB, and Malaria Programs: The Interaction of Health and Laboratory Systems, Clinical Research, and Service Delivery. American Journal of Clinical Pathology, 131, 849-851. https://doi.org/10.1309/AJCPGH89QDSWFONS

[15] Peter, T.F., Shimada, Y., Freeman, R.R., Ncube, B.N., Khine, A.A. and Murtagh, M.M. (2009) The Need for Standardization in Laboratory Networks. American Jour- 
nal of Clinical Pathology, 131, 867-874.

https://doi.org/10.1309/AJCPCBMOHM7SM3PI

[16] Magwai, T., Warasally, Z., Naidoo, N. and Gounden, V. (2020) Reducing Sample Rejection in Durban, South Africa. Clinical Chemistry and Laboratory Medicine, 59, 687-692. https://doi.org/10.1515/cclm-2020-0827

[17] Plebani, M. (2006) Errors in Clinical Laboratories or Errors in Laboratory Medicine? Clinical Chemistry and Laboratory Medicine, 44, 750-759.

https://doi.org/10.1515/CCLM.2006.123 